\title{
LDLR promoter variant and exon 14 mutation on the same chromosome are associated with an unusually severe $\mathrm{FH}$ phenotype and treatment resistance
}

\author{
Christine LH Snozek ${ }^{1,4}$, Susan A Lagerstedt ${ }^{1,4}$, Teck K Khoo ${ }^{2}$, Melvyn Rubenfire ${ }^{3}$, \\ William L Isley ${ }^{2,5}$, Laura J Train ${ }^{1}$ and Linnea M Baudhuin ${ }^{*}{ }^{\prime}$
}

\begin{abstract}
${ }^{1}$ Department of Laboratory Medicine and Pathology, Cardiovascular Laboratory Medicine, Mayo Clinic, Rochester, MN, USA; ${ }^{2}$ Division of Endocrinology, Diabetes, Metabolism, and Nutrition, Mayo Clinic, Rochester, MN, USA; ${ }^{3}$ Division of Cardiovascular Medicine, Department of Internal Medicine, University of Michigan, Ann Arbor, MI, USA
\end{abstract}

Familial hypercholesterolemia (FH) is the most common form of autosomal-dominant hypercholesterolemia, and is caused by mutations in the low-density lipoprotein receptor (LDLR) gene. Heterozygous FH is characterized by elevated low-density lipoprotein (LDL) cholesterol and early-onset cardiovascular disease, whereas homozygous FH results in more severe LDL cholesterol elevation with death by 20 years of age. We present here the case of an African-American female FH patient presenting with a myocardial infarction at the age of 48 , recurrent angina pectoris and numerous coronary artery stents. Her pretreated LDL cholesterol levels were more typical of a homozygous FH pattern and she was resistant to conventional lipid-lowering treatment, yet her other clinical parameters were not necessarily consistent with homozygous $\mathrm{FH}$. Genetic testing revealed two LDLR variants on the same chromosome: one a novel missense mutation in exon 14 (Cys681Gly) and the other a promoter variant (IVS1-217C $>$ T) previously shown to result in increased $L D L R$ transcription. Disease-associated PCSK9 or APOB mutations were not identified in this individual. Overall, her genetic and clinical profile suggests that enhanced expression of the mutant $L D L R$ allele resulted in a severe phenotype with characteristics of both heterozygous and homozygous FH.

European Journal of Human Genetics (2009) 17, 85-90; doi:10.1038/ejhg.2008.138; published online 23 July 2008

Keywords: familial hypercholesterolemia; conversion technology; LDLR; APOB; PCSK9

Introduction

Familial hypercholesterolemia (FH) is an autosomal dominant disorder of cholesterol regulation caused by mutations in the low-density lipoprotein receptor $(L D L R)$

\footnotetext{
*Correspondence: Dr LM Baudhuin, Laboratory Medicine and Pathology, Mayo Clinic, Hilton 402, 200 First St SW, Rochester, MN 55905, USA. Tel: + 1507284 2511; Fax: + 1507266 4176;

E-mail: baudhuin.linnea@mayo.edu

${ }^{4}$ These authors contributed equally to this work.

${ }^{5}$ In memoriam.

Received 10 December 2007; revised 20 June 2008; accepted 26 June 2008; published online 23 July 2008
}

gene. ${ }^{1}$ Germline impairment of LDLR function leads to lifelong, excessive levels of LDL cholesterol, resulting in tendinous xanthomata, atherosclerosis, and early-onset cardiovascular disease $(\mathrm{CVD}){ }^{2}$ Heritable mutations in other genes (APOB, PCSK9) whose products are involved in LDL regulation cause phenotypes resembling $\mathrm{FH}$. Disorders of excessive LDL because of mutations in $L D L R$, $A P O B$, and PCSK9 are categorized together as autosomal dominant hypercholesterolemia $(\mathrm{ADH})$, with a heterozygous incidence of approximately 1 in 500 in the general population. ${ }^{3,4}$ Heterozygous ADH typically results in LDL cholesterol levels above $200 \mathrm{mg} / \mathrm{dl}$, with the development 
of symptomatic CVD between the ages of 30 and 40 years. Homozygous or compound heterozygous $\mathrm{ADH}$ produces a much more severe phenotype, with LDL cholesterol often greater than $500 \mathrm{mg} / \mathrm{dL}$ and death before age 20 years. ${ }^{1}$

Treatment for $\mathrm{ADH}$ is aimed at lowering the plasma level of LDL and increasing LDL receptor activity. Heterozygotes are often treated with 3-hydroxy-3-methylglutaryl CoA reductase inhibitors (ie, statins), either in monotherapy or in combination with other drugs such as nicotinic acid and inhibitors of intestinal cholesterol absorption. Such drugs are generally not effective in FH homozygotes, and treatment in homozygotes or severe heterozygotes may consist of LDL apheresis, portacaval anastomosis, and liver transplantation. ${ }^{5,6}$

\section{Materials and methods Subject}

This study was approved by the Institutional Review Board at the Mayo Clinic and informed consent was obtained as required from the study participant.

\section{LDLR sequencing}

The promoter and all 18 exons of LDLR (GenBank NT_011295.10) and a minimum of 20 basepairs (bp) of intronic DNA flanking each exon were amplified by multiplexed polymerase chain reactions (PCR), with amplicons ranging in size from $278-755 \mathrm{bp}$. Amplification was performed using a common master mix containing Platinum Taq DNA Polymerase, 10X PCRx Enhancer System, 10X PCR Buffer (-MgCl), $\mathrm{MgSO}_{4}$ (all Invitrogen, Carlsbad, CA, USA), and a $10 \mathrm{~mm}$ dNTP mixture (Roche, Indianapolis, IN, USA). Master mix, forward and reverse primers were combined with genomic DNA and amplified by 35 cycles of PCR. Amplicons were bidirectionally sequenced using Big Dye Terminator technology on an ABI 3730 system (Applied Biosystems, Foster City, CA, USA). Sequence analysis was done using Mutation Surveyor $^{\mathrm{TM}}$ software (SoftGenetics, State College, PA, USA) and visual inspection.

\section{PCSK9 sequencing}

All 12 exons of PCSK9 (GenBank NM_174936) and a minimum of $20 \mathrm{bp}$ of intronic DNA flanking each exon were amplified through PCR, with amplicons ranging in size from 342 to $536 \mathrm{bp}$. Amplification and subsequent analysis were performed as detailed for $L D L R$.

\section{APOB genotyping}

$A P O B$ was genotyped for the two most common mutations associated with ADH: R3500W and R3500Q. Primers flanking $A P O B$ codon 3500 were used in a 30-cycle PCR. Amplicons were subjected to allele-specific primer extension incorporating biotinylated dCTP (Roche) and products were hybridized to fluorescent Flexmap beads (Luminex Corp, Austin, TX, USA). The biotin was conjugated to a Streptavidin, R-Phycoerythrin reporter solution (Molecular Probes, Eugene, OR, USA). Excess reporter solution was washed away from the beads with $1 \mathrm{X}$ hybridization buffer $(0.2 \mathrm{M} \mathrm{NaCl}, 0.1 \mathrm{M}$ Tris, $0.08 \%$ Triton X-100, $\mathrm{pH}$ 8.0) and samples were loaded into a Luminex $^{\circledR} 100$ for detection. Median fluorescence intensity signals were analyzed to determine allelic ratios (mutant signal/total signal) and genotype calls.

\section{LDLR large genomic rearrangement analysis}

Testing for large rearrangements of the $L D L R$ gene was done by use of a multiplex ligation-dependent probe amplification kit (MRC Holland, Amsterdam, the Netherlands), according to the manufacturer's instructions. Briefly, genomic DNA was hybridized to probe pairs targeted to the $18 L D L R$ exons and the proximal promoter. After a 16-h hybridization, probes were ligated and amplified by PCR. Detection was performed using capillary electrophoresis on an ABI 3130xl (Applied Biosystems). LDLR target peak heights were normalized to internal control targets and assessed for exon loss (eg, a relative gene ratio of 0.5 for a single copy deletion) or exon gain (eg, a gene ratio of 1.5 for a single copy gain) using GeneMarker software v1.41 (SoftGenetics).

\section{Conversion analysis}

Determination of whether the $L D L R$ variants were present on the same copy (cis) or opposite copies (trans) of chromosome 19 was performed by conversion technology (CT) in the Mayo Clinic Conversion Technology Laboratory. CT is a method of developing human-mouse somatic cell hybrids for the purpose of isolating human chromosomes. ${ }^{7}$ Briefly, patient lymphocytes were fused with the mouse E2 cell line and human-mouse hybrids were then selected in culture medium containing G418, hypoxanthine, aminopterin, and thymidine. After selection, hybrids containing human chromosome 19 were identified by fluorescence in situ hybridization using five independent microsatellite markers (D19S216, D19S221, D19S566, D19S220, and D19S210). Clones containing a haploid copy of human chromosome 19 were isolated for DNA, which was then subjected to sequencing for the $L D L R$ promoter and exon 14 variants.

\footnotetext{
Results

A female patient of African-American descent was referred to our institution at age 49 for treatment-refractory hyperlipidemia. At the age of 48 years, 18 months prior to the referral, the patient had suffered a myocardial infarction at which time it was noted that her LDL cholesterol was severely elevated (anecdotally as high as $>1000 \mathrm{mg} / \mathrm{dl}$ ). The patient had recurrent angina pectoris, an abnormal nuclear stress test, and at the time of consult, had received 14 coronary artery stents. She did not use
} 
Table 1 Hypercholesterolemia-related gene variants found in this patient

\begin{tabular}{|c|c|c|c|}
\hline Gene & Nucleotide change & Amino acid affected & Significance (heterozygous frequency ${ }^{\mathrm{a}}$ ) \\
\hline$L D L R$ & $2041 T>G$ & Cys681Gly & Likely pathogenic; similar FH variants known \\
\hline$L D L R$ & IVS1-217C >T & $\mathrm{n} / \mathrm{a}$ (promoter) & Enhances expression to $\sim 160 \%$ in vitro \\
\hline PCSK9 & $1420 \mathrm{~A}>\mathrm{G}$ & Ile474Val & Occurs with both high and low LDL $(0.217-0.441)$ \\
\hline PCSK9 & IVS658-7C $>T$ & $\mathrm{n} / \mathrm{a}$ (intronic) & Benign variant $(0.403-0.542)$ \\
\hline PCSK9 & IVS799+3G $>A$ & $\mathrm{n} / \mathrm{a}$ (intronic) & Benign variant $(0.435)$ \\
\hline PCSK9 & $1380 A>G$ & Val460Val & Variant of unknown significance $(0.217-0.441)$ \\
\hline
\end{tabular}

$\mathrm{FH}$, familial hypercholesterolemia; LDL, low-density lipoprotein cholesterol.

a PCSK9 heterozygous frequencies as reported for African or African-American populations on the NCBI SNP website: www.ncbi.nlm.nih.gov/SNP/.

nicotine or ethanol, ate an excellent diet with minimal meat and dairy intake, had no history of diabetes mellitus, and was adopted with family history unknown. Upon physical exam, this individual demonstrated to be moderately overweight (BMI 26.8) with a small goiter. No tendon xanthomata or other outward signs of hypercholesterolemia were visible initially, though subsequent examination revealed Achilles tendon xanthomata.

At the time of presentation to our institution, her LDL and total cholesterol levels were markedly high at 393 and $483 \mathrm{mg} / \mathrm{dl}$, respectively, despite therapy with maximumdose simvastatin/ezetimibe, colesevelam, and fish oil. Several previous treatment regimens (atorvastatin, rosuvastatin, rosuvastatin plus ezetimibe, and atorvastatin/ ezetimibe plus colesevelam) had similarly failed to reduce her LDL cholesterol significantly. Weekly LDL apheresis was instituted and resulted in a reduction of her LDL cholesterol to, at its lowest, $251 \mathrm{mg} / \mathrm{dl}$. The patient had difficulties with recurrent vascular access infections that precluded routine LDL apheresis; however, when therapy was possible, the rate of LDL rebound after apheresis was quite rapid, consistent with rates seen in homozygous $\mathrm{FH}^{8}$ Throughout 2 years of treatment, the patient's LDL cholesterol ranged between 251 and $569 \mathrm{mg} / \mathrm{dl}$, with total cholesterol between 328 and $625 \mathrm{mg} / \mathrm{dl}$.

The patient's triglyceride levels were high $(282 \mathrm{mg} / \mathrm{dl})$ at initial presentation. Without medical therapy directed at triglycerides or VLDL particles (although the patient did convert to a vegetarian diet), the triglyceride level subsequently decreased to $\leq 160 \mathrm{mg} / \mathrm{dl}$. Other lipid results were abnormal, including a highly variable low level of high-density lipoprotein cholesterol (ranging between $24-47 \mathrm{mg} / \mathrm{dl}$ ), and mildly elevated lipoprotein(a). Fasting glucose was $103 \mathrm{mg} / \mathrm{dl}$ and TSH was within the reference range, making undiagnosed diabetes mellitus or hypothyroidism unlikely as causes of the dyslipidemia. She had low IGF-1 and was therefore suspected to have growth hormone (GH) deficiency; however, the mild elevation in LDL cholesterol associated with GH deficiency is unlikely to cause the remarkably high cholesterol levels seen in this patient. $^{9}$

Given the patient's history and lipid levels, molecular testing for genes related to ADH was pursued. Full-gene sequencing for $L D L R$ uncovered two point variants (Table 1): a novel exon 14 mutation (2041T > G; Cys681Gly) and a promoter region variant (IVS1-217C $>\mathrm{T}$ ) that has previously been linked to increased transcription of $L D L R .{ }^{10}$ Gene dosage analysis of $L D L R$ by multiplex ligation-dependent probe amplification did not reveal any large rearrangements in the gene. Similarly, the patient was negative for the two most common disease-causing mutations in $A P O B$. Sequencing of PCSK9 revealed four heterozygous variations from wild-type sequence (Table 1). One of these (Ile474Val) has been linked to dysregulation of LDL cholesterol, however a large study in African Americans showed that this variant can occur with both high and low LDL cholesterol. ${ }^{11}$ The other three PCSK9 variants have been reported previously as polymorphisms or variants that are likely not linked to LDL cholesterol levels. ${ }^{12}$

The presence of two $L D L R$ variants led to the question of whether the variants were present on the same chromosome (ie, in cis) or on different chromosomes (ie, in trans). As the patient was adopted and had no knowledge of her biological parents or other family members, family studies could not be pursued. Therefore, to establish the chromosomal location of the two identified $L D L R$ variants, conversion technology was used to separate the two copies of chromosome 19. Sequencing of the DNA from each haploid copy of chromosome 19 for the $L D L R$ exon 14 and promoter variants revealed that both variants were present on the same haploid chromosome (ie, in cis).

\section{Discussion}

We present here the intriguing case of an $\mathrm{FH}$ individual with an unusual phenotype. The patient's untreated LDL cholesterol levels were in a range most typical of homozygous $\mathrm{FH}$, yet such patients rarely live beyond the age of 20. In addition, the patient's cholesterol levels were resistant to an excellent diet (low cholesterol and very low saturated fat) and to all available cholesterol-lowering therapies; LDL rebound after apheresis was similar to rates expected with homozygous disease. However, she had only small Achilles xanthomata with no other external features 
of classical FH, apart from the persistently elevated LDL cholesterol.

Diabetes mellitus and thyroid dysfunction were ruled out as potential causes for dyslipidemia. The suspected GH deficiency may have played some role; however, the effect of $\mathrm{GH}$ on cholesterol regulation is likely insufficient to explain the degree of hypercholesterolemia seen in this patient. Growth hormone replacement therapy results in a $10-20 \%$ decline in total and LDL cholesterol in non-FH patients; ${ }^{13}$ even if this degree of improvement were the same in the setting of $\mathrm{FH}$, it would not reduce this patient's lipids to the level expected for an FH heterozygote. GH therapy in non-GH-deficient $\mathrm{FH}$ heterozygotes did not significantly reduce lipid levels, ${ }^{14}$ suggesting that the GH pathway likely has a lower relative effect in $\mathrm{FH}$ than in non-FH patients. Thus it is probable that this patient's suspected GH deficiency was only a minor contributor to the overall dyslipidemia.

Similarly, the patient's oophorectomy and hysterectomy at age 36 may have exacerbated her hypercholesterolemia; menopause has been shown to increase the risk of CVD in women, presumably through loss of estrogen-mediated protection. ${ }^{15}$ However, though the number of studies addressing the effect of menopause in $\mathrm{FH}$ patients is limited, results suggest that total cholesterol is only slightly higher in postmenopausal $\mathrm{FH}$ heterozygotes. ${ }^{16}$ Interestingly, the effect of menopause seems to be visible in both cholesterol and triglyceride levels, which may in part account for this patient's initial hypertriglyceridemia.

Testing of the three major genes linked to ADH revealed two $L D L R$ variants on the same chromosome, but no other genetic variants likely to result in hypercholesterolemia. As testing for $A P O B$ variants other than R3500W and R3500Q was not performed, the presence of other $A P O B$ mutations in this individual cannot be ruled out. In addition, there may be other genes capable of modifying LDL regulation that were not analyzed. ${ }^{17}$

The patient was later found to be homozygous for the E4 variant of $A P O E$, encoding apolipoprotein E. This genotype has been linked to elevations in both cholesterol and triglycerides, suggesting that APOE may contribute to this patient's phenotype. However, studies show that the E4 allele confers less additional risk ${ }^{17}$ or resistance to statin therapy $^{18}$ in $\mathrm{FH}$ heterozygotes as compared with the general population, suggesting that $A P O E$ is likely not sufficient to explain the massive dyslipidemia and refractoriness in this patient. In addition, FH heterozygotes who are E4 carriers show a trend toward enhanced LDL lowering in response to diet, relative to carriers of either E3 or E2. ${ }^{19}$ Thus, given this patient's long-term adherence to an excellent diet, her $A P O E$ status may have actually contributed to a better lipid profile than would be expected with a standard diet or different $A P O E$ genotype.

Although PCSK9 is involved only rarely in $\mathrm{ADH}$, genetic PCSK9 variants that are associated with altered regulation of LDL cholesterol levels are frequently found in the African-American population. ${ }^{20}$ In addition, PCSK9 mutation has been linked to accelerated ApoB production, ${ }^{21}$ and can worsen the phenotype of heterozygous $\mathrm{FH}^{22}$ One of the PCSK9 variants we found in this patient was previously, though not definitively, linked to alterations in cholesterol levels; it was initially associated with elevated cholesterol, but a subsequent, larger study in African Americans linked it with both unusually high and unusually low LDL cholesterol values. ${ }^{11}$ It is possible that the effect of PCSK9 alteration varies by context: the previously reported mutant may not sufficiently alter cholesterol regulation to cause a noticeable phenotype in isolation, but is capable of worsening dyslipidemia when combined with defective LDLR. Unfortunately, the exact details of how PCSK9 affects LDLR and cholesterol regulation remain incompletely characterized, thus further studies are needed to elucidate the importance of this PCSK9 variant in the setting of $\mathrm{FH}$.

Alternatively, we hypothesize that the combination of the two $L D L R$ variants is responsible for the severity of this patient's disease. Although the Cys681Gly mutation has not been previously reported, it is likely to disrupt LDLR function for several reasons: (1) the mutation is a disfavored substitution of an evolutionarily conserved residue; (2) the mutation occurs in the EGF precursor homology domain which is required for positioning the LDL-binding domain; ${ }^{6}$ and (3) other missense and nonsense mutations at this residue (C681X, C681Y, and C681S) have been previously characterized as disease-causing. ${ }^{23-25}$ However, the previously described mutations at residue 681 have not been associated with exceptionally uncharacteristic FH phenotypes. The IVS1-217C $>$ T promoter variant has been shown in vitro to increase $L D L R$ transcription to $\sim 160 \%$ of normal. ${ }^{10}$ Because the promoter variant is in cis with the exon 14 mutation, there would be an expected increase in expression of the mutant Cys681Gly allele. Thus, enhanced transcription of the mutated receptor likely shifts the balance of expression to favor that of the mutant $L D L R$ gene relative to the wild type, resulting in a gene-dosage effect leading to a unique phenotype that has features of both classic heterozygous and classic homozygous $\mathrm{FH}$.

Although this patient's Cys681Gly mutation is novel, a previous report of a base change at the same nucleotide position provides support for the importance of the cis promoter variant. Sun et $a^{24}$ describe a $2041 \mathrm{~T}>$ A transversion resulting in a Cys681Ser amino-acid alteration (published as Cys660Ser because of an older numbering system). Interestingly, despite a similar amino-acid change, the Cys681Ser patient had pretreatment cholesterol levels (LDL $390 \mathrm{mg} / \mathrm{dl}$, total $448 \mathrm{mg} / \mathrm{dl}$ ) that were not nearly as elevated as our patient's pretreatment levels. Furthermore, the patient in the Sun et $a l^{24}$ report responded well to simvastatin/ezetimibe, with an on-treatment LDL 
cholesterol of $150 \mathrm{mg} / \mathrm{dl}$. On the other hand, without concomitant apheresis, neither simvastatin/ezetimibe nor several other statin-based therapies successfully reduced our patient's LDL cholesterol.

The degree of hyperlipidemia in this patient and her resistance to statin therapy are unusual but not unique in the spectrum of FH presentations. There are reports of treated ${ }^{26}$ and untreated ${ }^{27}$ homozygous FH patients living well beyond the expected lifespan; however, whether the patients in these reports had genetically confirmed homozygous $\mathrm{FH}$ is unclear. In contrast, one intriguing case report described an FH patient with a myocardial infarction at age 26 and markedly elevated, statin-resistant LDL cholesterol; the patient had two heterozygous LDLR mutations in exons 6 and 9 in cis. $^{5}$ Although the genetics of that case report are different than the patient presented here, both cases nonetheless support the concept of an unusually severe 'heterozygous' FH phenotype associated with dual variants in a single $L D L R$ allele.

The unique molecular genetic configuration described here brings to light the importance of thorough analysis when attempting to understand uncharacteristic genetic phenotypes and optimize clinical care. Although this case is the first report of its kind, future studies may help clarify the frequency of the promoter variant and whether or not its presence in cis with other disease-causing mutations results in a similar FH phenotype. This could potentially lead to a 'pharmacogenetic' approach to FH in that when encountering the presence of this promoter variant in combination with a disease-causing FH mutation, one might consider implementing more aggressive LDLlowering therapies at an earlier stage.

Although studies have shown a link between the severity of $L D L R$ mutations and the clinical course, there remains much to learn about FH genotype-to-phenotype correlations both in clinical presentation ${ }^{28}$ and in the response to statin therapy. ${ }^{29}$ Some studies show better statin efficacy in patients with severe $L D L R$ mutations, whereas others show no difference or the opposite result. Unfortunately, work in this area is hampered by the large number of $L D L R$ variants and the substantial influence of environmental, dietary, and other genetic factors. This report re-emphasizes the need for a thorough analysis of genes associated with ADH to clarify the influence of multiple pathogenic mutations.

\section{References}

1 Garg A, Simha V: Update on dyslipidemia. J Clin Endocrinol Metab 2007; 92: 1581-1589.

2 Sibley C, Stone NJ: Familial hypercholesterolemia: a challenge of diagnosis and therapy. Cleve Clin J Med 2006; 73: 57-64.

3 Soutar AK, Naoumova RP: Mechanisms of disease: genetic causes of familial hypercholesterolemia. Nat Clin Pract Cardiovasc Med 2007; 4: 214-225.

4 Austin MA, Hutter CM, Zimmern RL, Humphries SE: Genetic causes of monogenic heterozygous familial hypercholesterolemia: a HuGE prevalence review. Am J Epidemiol 2004; 160: 407-420.

5 Alkofer BJ, Chiche L, Khayat A et al: Liver transplant combined with heart transplant in severe heterozygous hypercholesterolemia: report of the first case and review of the literature. Transplant Proc 2005; 37: 2250-2252.

6 Goldstein JL, Hobbs HH, Brown MS: Familial hypercholesterolemia; in: Scriver CR, Beaudet AL, Valle D, Sly WS (eds): Metabolic and Molecular Bases of Inherited Disease. New York: McGraw Hill, 2001, pp.1-122, chapter 120.

7 Papadopoulos N: Conversion technology and its role in genetic testing of inherited diseases. Expert Rev Mol Diagn 2003; 3: 497-506.

8 Thompsen J, Thompson PD: A systematic review of LDL apheresis in the treatment of cardiovascular disease. Atherosclerosis 2006; 189: $31-38$.

9 Monson JP: Long-term experience with GH replacement therapy: efficacy and safety. Eur J Endocrinol 2003; 148 (Suppl 2): S9-S14.

10 Scholtz CL, Peeters AV, Hoogendijk CF et al: Mutation -59c $\rightarrow \mathrm{t}$ in repeat 2 of the LDL receptor promoter: reduction in transcriptional activity and possible allelic interaction in a South African family with familial hypercholesterolaemia. Hum Mol Genet 1999; 8: 2025-2030.

11 Kotowski IK, Pertsemlidis A, Luke A et al: A spectrum of PCSK9 alleles contributes to plasma levels of low-density lipoprotein cholesterol. Am J Hum Genet 2006; 78: 410-422.

12 Chen SN, Ballantyne CM, Gotto Jr AM, Tan Y, Willerson JT, Marian AJ: A common PCSK9 haplotype, encompassing the E670G coding single nucleotide polymorphism, is a novel genetic marker for plasma low-density lipoprotein cholesterol levels and severity of coronary atherosclerosis. J Am Coll Cardiol 2005; 45: $1611-1619$.

13 Burger AG, Monson JP, Colao AM, Klibanski A: Cardiovascular risk in patients with growth hormone deficiency: effects of growth hormone substitution. Endocr Pract 2006; 12: 682-689.

14 Tonstad S, Sundt E, Ose L et al: The effect of growth hormone on low-density lipoprotein cholesterol and lipoprotein (a) levels in familial hypercholesterolemia. Metabolism 1996; 45: 1415-1421.

15 Seed M: The choice of hormone replacement therapy or statin therapy in the treatment of hyperlipidemic postmenopausal women. Atheroscler Suppl 2002; 3: 53-63.

16 Kolovou GD, Anagnostopoulou KK, Pilatis ND et al: The influence of natural menopause on postprandial lipemia in heterozygotes for familial hypercholesterolemia. J Womens Health (Larchmt) 2004; 13: 1119-1126.

17 Bertolini S, Pisciotta L, Di Scala L et al: Genetic polymorphisms affecting the phenotypic expression of familial hypercholesterolemia. Atherosclerosis 2004; 174: 57-65.

18 Christidis DS, Liberopoulos EN, Kakafika AI et al: The effect of apolipoprotein E polymorphism on the response to lipid-lowering treatment with atorvastatin or fenofibrate. I Cardiovasc Pharmacol Ther 2006; 11: 211-221.

19 Carmena-Ramon R, Real JT, Ascaso JF, Ordovas JM, Carmena R: Effect of apolipoprotein E genotype on lipid levels and response to diet in familial hypercholesterolemia. Nutr Metab Cardiovasc Dis 2000; 10: 7-13.

20 Cohen J, Pertsemlidis A, Kotowski IK, Graham R, Garcia CK, Hobbs HH: Low LDL cholesterol in individuals of African descent resulting from frequent nonsense mutations in PCSK9. Nat Genet 2005; 37: 161-165.

21 Ouguerram K, Chetiveaux M, Zair Y et al: Apolipoprotein B100 metabolism in autosomal-dominant hypercholesterolemia related to mutations in PCSK9. Arterioscler Thromb Vasc Biol 2004; 24: $1448-1453$.

22 Pisciotta L, Priore Oliva C, Cefalu $\mathrm{AB}$ et al: Additive effect of mutations in LDLR and PCSK9 genes on the phenotype of familial hypercholesterolemia. Atherosclerosis 2006; 186: $433-440$.

23 Lehrman MA, Schneider WJ, Brown MS et al: The Lebanese allele at the low density lipoprotein receptor locus. Nonsense mutation 
produces truncated receptor that is retained in endoplasmic reticulum. J Biol Chem 1987; 262: 401-410.

24 Sun XM, Patel DD, Knight BL, Soutar AK: Influence of genotype at the low density lipoprotein (LDL) receptor gene locus on the clinical phenotype and response to lipid-lowering drug therapy in heterozygous familial hypercholesterolaemia. The familial hypercholesterolaemia regression study group. Atherosclerosis 1998; 136: 175-185.

25 Schuster H, Keller C, Wolfram G, Zollner N: Ten LDL receptor mutants explain one third of familial hypercholesterolemia in a German sample. Arterioscler Thromb Vasc Biol 1995; 15: $2176-2180$.
26 Francis GA, Johnson RL, Findlay JM, Wang J, Hegele RA: Cerebral cholesterol granuloma in homozygous familial hypercholesterolemia. CMAJ 2005; 172: 495-497.

27 Archer SL, Paterson I, Francis GA: Images in cardiovascular medicine. Untreated 37-year-old homozygous familial hypercholesterolemic smoker. Circulation 2006; 113: e777.

28 Nicholls P, Young IS, Graham CA: Genotype/phenotype correlations in familial hypercholesterolaemia. Curr Opin Lipidol 1998; 9: $313-317$.

29 Choumerianou DM, Dedoussis GV: Familial hypercholesterolemia and response to statin therapy according to LDLR genetic background. Clin Chem Lab Med 2005; 43: 793-801. 